\title{
Mycobacteria fecal shedding in wild boars (Sus scrofa), South-eastern France
}

2

3

Mustapha Fellag ${ }^{1}$, Michel Drancourt ${ }^{1,{ }^{*}}$, Jean-Lou Marié ${ }^{2}$, Bernard Davoust ${ }^{1,2}$

$4 \quad{ }^{1}$ Aix-Marseille Université, MEPHI, IRD, IHU Méditerranée Infection, Marseille,

5 France.

$6 \quad{ }^{2}$ Animal Epidemiology Working Group of the Military Health Service, DRSSA Toulon,

7 France.

$8 \quad{ }^{*}$ Corresponding author: Prof. Michel Drancourt

$9 \quad$ E-mail: michel.drancourt@mediterranee-infection.fr 
11

12

\section{ABSTRACT}

The recent recrudescence of tuberculosis in cattle has implicated wild boar as a reservoir and vector of this disease, which led to the investigation of fecal shedding of the causative Mycobacterium bovis. In the Provence region of France, wild boars are very abundant, and the current study was carried out to assess the presence of tuberculous and nontuberculous mycobacteria in feces of wild boar population. W e developed an original protocol allowing the fast isolation of mycobacteria by combining a 1\%-chlorhexidine decontamination of fecal matter with culture on MOD9 medium. Colonies were identified by matrix-assisted laser desorption/ionization timeof-flight mass spectrometry, combined with DNA sequencing. This protocol yielded no tuberculous mycobacteria among ninety-nine wild boar fecal samples collected in the Provence region. However, non-tuberculous mycobacteria were isolated from five samples (5.05\%), including Mycobacterium peregrinum, Mycobacterium vaccae and Mycobacterium setense, the last species being previously unreported in the wild boar; in addition to two positive samples for Nocardia spp. In conclusion, wild boars in southeastern France are not shedding tuberculosis agents, but they could constitute a reservoir of human non-tuberculous mycobacteriosis in selected populations directly exposed to wild boars. 


\section{INTRODUCTION}

Bovine tuberculosis is a zoonosis due to Mycobacterium bovis (M. bovis) affecting both livestock and wild mammals ${ }^{1}$. Bovine tuberculosis is a contagious infection of significant health and economic importance and eradication programs have been implemented in a number of developed countries ${ }^{1,2}$. These programs yielded a remarkable reduction of the incidence of the disease ${ }^{1,3}$.

However, the wide distribution of $M$. bovis in wildlife is a major obstacle to the eradication of tuberculosis in cattle ${ }^{2,3}$. In numerous countries, bovine tuberculosis is maintained by multi-host systems involving cattle and wild mammals ${ }^{2-4}$. While the role of the red deer (Cervus elaphus) remains controversial ${ }^{3}$, the European badger (Meles meles) in Great Britain and Ireland and the wild boar (Sus scrofa) in the Iberian Peninsula are acknowledged reservoirs for $M$. bovis ${ }^{3,5}$. The later species is also recognized as a host for other Mycobacterium tuberculosis complex, including Mycobacterium microti ${ }^{6}$ and Mycobacterium caprae ${ }^{7}$. In addition, non-tuberculous mycobacteria that are widely distributed in the environment are not uncommon in wild boars, where they were isolated from lymph nodes ${ }^{8}$ and feces ${ }^{9,10}$. These nontuberculous mycobacteria have been identified as emerging human pathogens, especially in immunocompromised patients ${ }^{11,12}$.

Accordingly, naturally infected wild boars excrete $M$. bovis mycobacteria by oronasal and digestive routes, with inocula above $10^{3}$ colony-forming units (CFU)/g ${ }^{13}$. Furthermore, it has been shown that $M$. bovis can persist in soil for several months ${ }^{14}$. Interactions between $M$. bovis and soil-inhabiting amoebae may increase the lifespan of mycobacteria, thus promoting their transmission throughout the environment ${ }^{15}$. Humans and animals may then be contaminated not only by direct contact with 
infected wild animals, as is the case for hunters handling carcasse ${ }^{16}$, but also from a contaminated environment ${ }^{9}$.

For epidemiological investigations and the diagnosis of tuberculosis in wild animals, many techniques have been developed, including immunological, serological and molecular biology techniques but culture remains the gold standard technique for the diagnosis of tuberculosis mycobacteria infections ${ }^{17}$. The lymph nodes are the most frequently used sample for the research of mycobacteria in animals ${ }^{17-19}$ but this invasive sampling made by well trained staff, is almost limited to dead animals. Therefore, the analysis of feces is an emerging method in wild animals 13,20-22 on the model of what has been reported for the routine diagnosis in human patients ${ }^{23}$.

In France, the first cases of tuberculosis in wild animals were detected in 2001 in the Brotonne forest, Normandy ${ }^{2}$. Then, monitoring programs for tuberculosis in wild animals were applied and $M$. bovis was detected in badgers, wild boars, red deer, roe deer and red fox in proximity areas of bovine tuberculosis outbreak ${ }^{4,19,22,24}$. One case of tuberculosis was detected in a wild boar in Loir-et-Cher county which had remained free from bovine tuberculosis for more than twenty years ${ }^{25}$. In Southeastern France, there is a lack of information regarding the presence of tuberculosis and nontuberculous mycobacteria in wild boars, except for information from the national surveillance system for tuberculosis in free-ranging wildlife (Sylvatub) which indicates that wild animals collect in this area are negative ${ }^{4}$ whereas one study carried-out by serological methods that indirectly detected cases of $M$. bovis $^{26}$.

Here, we investigated the presence and the shedding of Mycobacterium tuberculosis complex and nontuberculous mycobacteria in feces of wild boars living 
in Provence region, France by using an original decontamination and culture protocol.

\section{Results}

Artificially-infected feces samples. Using $\mathrm{NA}-\mathrm{OH}$ decontamination and culture on Coletsos medium, PBS negative controls remained sterile whereas culture of noninoculated feces samples were invaded by contaminants. As for feces artificially inoculated with $M$. bovis BCG for ten days, one culture was positive with seven colonies of $M$. bovis BCG confirmed by MALDI-TOF-MS in addition to contaminants, the other two cultures were invaded by contaminants without presence colonies of $M$. bovis BCG. The contaminants were identified by MALDI-TOF-MS as various Bacillus species. Using chlorhexidine 1\% decontamination and culture on MOD9 medium, PBS negative controls and non-inoculated feces remained sterile. As for feces artificially inoculated with $10^{5}$ CFUs $M$. bovis BCG, non-frozen feces cultures yielded the first colonies of $M$. bovis BCG after seven and nine days of incubation and more than 100 colonies were observed after 20 days of incubation in one plate and 37 colonies in the second culture plate. Only one Sphingomonas paucimobilis contaminant was observed on one of the two cultures. Feces artificially contaminated and frozen at $-20^{\circ} \mathrm{C}$ yielded M. bovis BCG colonies after nine-day incubation and after twenty-day incubation and 12 colonies were counted in one plate and only four on the second culture plate, in the absence of any contaminant. These data indicate a sensitivity of $10^{3}$ CFUs (fresh feces) and $10^{4}$ CFUs (frozen feces) on MOD9 medium. This method of culture confirmed that feces artificially contaminated with BCG were negative for mycobacteria.

Culture of wild boar feces. A total of 99 wild boar feces were decontaminated using $1 \%$ chlorhexidine and cultured on MOD9 medium. Colonies were observed after 4 to 
8 days of incubation. Cultures obtained after one-month incubation were classified into four types: plates containing pure cultures of mycobacteria $(n=1)$; plates containing mycobacteria mixed with contaminants $(n=4)$; plates containing only contaminants $(n=15)$ including two positive Nocardia spp. cultures; and negativeculture plates $(n=79)$. No further colony was observed after three additional months of incubation (for a total of four-month incubation). None of these 99 samples yielded M. bovis, but non-tuberculous mycobacteria were cultivated in five samples which corresponds to a rate of $5.05 \%$. Mycobacterium vaccae was isolated in one feces sample collected at military camp A, along with Mycobacterium peregrinumin in three samples and Mycobacterium setense in one sample, collected at military camp B. These mycobacteria were all identified by MALDI-TOF-MS and confirmed by partial rpoB gene sequencing (Table 1). In addition, Nocardia rhamnosiphila was identified by $16 \mathrm{~S}$ rRNA gene sequencing in one sample collected at military camp A and one Nocardia spp. isolate representative of a new Nocardia species in one sample collected in camp $\mathrm{B}^{27}$. MALDI-TOF-MS identified contaminants as Pseudomonas putida, Pandoraeasputorum, Ralstonia pickettii, Sphingomonas paucimobilis and Sphingobacterium faeciuma long with a non-identified fungus.

\section{DISCUSSION}

Culture-based investigation of wild boar feces collected in two geographic areas in the Provence region of France failed to find living tuberculous mycobacteria including M. bovis. This observation was made after an original protocol of feces decontamination and culture had been validated using the closely related $M$. bovis BCG strain, in the presence of negative controls which remained negative. 
126 Culture remains the reference method for the routine confirmation of mycobacterial

127 infection in animals ${ }^{1,28}$. The culture protocol herein described allows to detect an

128 inoculum of $10^{3}-10^{4}$ CFUs $M$. bovis which is in the range of that extrapolated from PCR-based observations in wild boars in Spain ${ }^{15}$. However, contamination is a major limitation of culturing feces. Here, we applied to animal feces a $1 \%$ chlorhexidine decontamination and MOD9 medium culture protocol suitable for the recovery of Mycobacterium tuberculosis complex in patients on diagnosed with pulmonary tuberculosis ${ }^{29,30}$. Because the level of contamination of swine feces is very high, an efficient decontamination protocol is essential to isolate and culture mycobacteria from this type of sample ${ }^{20}$. In a first step, we validated this protocol using artificially inoculated feces. Then, we applied it for the first time on animal specimens combined with MOD9 medium protocol and found that this protocol was indeed suitable for the recovery of living mycobacteria from wild boar feces. These results contrasted with the standard NALC-NaOH decontamination and culture on Coletsos medium, which yielded contaminants invading the culture tubes. feces compared to culturing fresh feces. The same observation was previously reported regarding the decreased viability of $M$. bovis after freezing ${ }^{13}$, whereas no effect of freezing at $-20^{\circ} \mathrm{C}$ was reported when culturing $M$. tuberculosis from sputum specimens collected in patients ${ }^{31}$. As for feces, the recommendation is to cultivate fresh feces collected in cattle within less than 96 hours for the isolation and culture of M. avium $^{21}$.

The $1 \%$ chlorhexidine decontamination and MOD9 culture protocol enabled the isolation of three non-tuberculous mycobacteria from five different wild boars with a prevalence of $5.05 \%$, in the range of the previously reported $0 \%$ to $11.1 \%$ prevalence 
of non-tuberculous mycobacteria in wild boar faeces ${ }^{10}$. In the lymph nodes, prevalence is $16.8 \%$ in Spain ${ }^{8}$ whereas no mycobacteria could be detected in lymph nodes in another study in Poland ${ }^{32}$ the prevalence of mycobacteria in wild boar varies among wild boar population, density and environment. $M$. vaccae was initially cultivated from cows in Austria ${ }^{33}$ and has previously been isolated in wild boars in Australia ${ }^{9}$. M. vaccae is often considered a non-pathogenic mycobacterium ${ }^{34}$ and has even been proposed as a vaccine for the treatment of multiresistant tuberculosis ${ }^{35}$. However, cases of pulmonary and skin infections have been described in human diseases ${ }^{12,33}$. Furthermore, rpoB gene sequencing confirmed the result obtained by MALDI-TOF and unambiguously ${ }^{36}$ distinguished $M$. peregrinum from the closely related $M$. septicum. Indeed, the species are not differentiated by 16S rRNA gene sequencing ${ }^{36}$. In animals, M. peregrinum has been described as an etiological agent of mycobacteriosis in farmed fish ${ }^{37}$ was recently isolated in wild boar from Slovenia ${ }^{18}$. It is an opportunistic pathogen previously implicated in surgical site infections and catheter-related infections ${ }^{12,38}$. Regarding $M$. setense isolated here in one wild boar from military camp $B$, it has been described for the first time as being responsible for osteitis ${ }^{39,40}$. Furthermore, three cases have been reported in Italy ${ }^{12}$ and Iran ${ }^{41}$. We report here on the first isolation of $M$. setense in wild boars. $M$. peregrinum and $M$. setense are two members of the Mycobacterium fortuitum complex, which is including rapidly growing non-tuberculous mycobacteria 40 distributed in the aquatic environment ${ }^{18,42}$. This may suggest the presence of these mycobacteria in the aquatic environments frequented by the wild boar at military camp B.

Moreover, the original culture protocol reported here allowed isolating two species of Nocardia. The first isolate was definitely identified as N. rhamnosiphila by $16 \mathrm{~S}$ 
rRNA gene sequencing. This species, initially identified from a compost mound sample in South Africa ${ }^{43}$ has never been reported in animals and not in the humans. The second isolate here referred as Nocardia strain S-137 is a representative of a hitherto undescribed species: this isolate exhibited a non-identifying MALDI-TOF-MS spectrum, along with a $16 \mathrm{~S}$ rRNA gene sequence exhibiting only $98 \%$ sequence similarity with $N$. brasiliensis strain FDAARGOS. Draft genome sequence and in silico DNA-DNA hybridization confirmed strain Nocardia S-137 as representative of a new species "Nocardia suismassiliense" 27.

While the general population is not routinely exposed to wild boars, some selected populations chiefly hunted, are exposed in such way that wild boars do constitute the reservoir of zoonoses, as illustrated by the demonstration of cross-transmission of hepatitis $E$ virus from wild boars to patients in our region ${ }^{44,45}$.

In conclusion, we found no evidence for living Mycobacterium tuberculosis complex, including M. bovis, in the feces of wild boars collected during 2015/2016 hunting season in the Provence region of France whereas other actinomycetes including non-tuberculous mycobacteria, were easily cultured. The decontaminationculture protocol reported here can be used for routine, culture-based detection of mycobacteria and related bacteria in wild boar feces samples, a source of previously undescribed microorganisms.

\section{Materials and methods}

Sample collection. In order to study naturally excreted mycobacteria in wild boar feces, a total of 99 fecal samples were collected during the $2015 / 2016$ hunting season in the Provence region (South-East of France). A total of 79 samples were

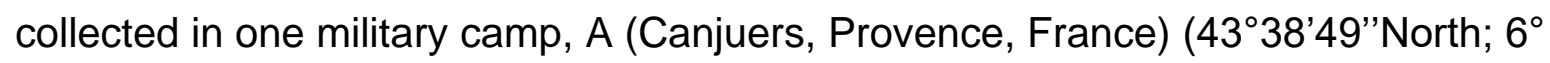


$27^{\prime} 56$ "East) and 20 samples in another military camp, B (Carpiagne, Provence,

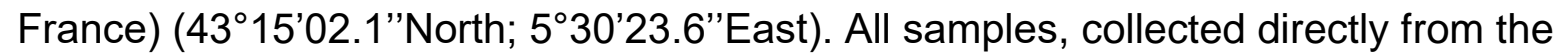
animal rectum by hunters were stored at $-20^{\circ} \mathrm{C}$ until cultured at the latest eight months after collection. No animals were killed specifically for this study and this work did not require animal experiment ethics approval.

Artificially contaminated feces specimens. M. bovis strain BCG was grown at $37^{\circ} \mathrm{C}$ on Middlebrook $7 \mathrm{H} 10$ agar (Becton Dickinson, Pont de Claix, France) supplemented with oleic-albumin-dextrose-catalase (OADC) (Becton Dickinson). The colonies were suspended in a phosphate buffered saline (PBS), then rigorously vortexed using 3-mm sterile glass beads (Sigma-Aldrich, Saint-Quentin-Fallavier, France) and passed five times through a 25-G needle to disperse clustered cells. The homogenized suspensions were then calibrated at $10^{7} \mathrm{CFU} / \mathrm{mL}$ by using optical density at $580 \mathrm{~nm}$ (Cell Density Meter; Fisher Scientific, Illkirch, France) and calibration curve. Feces of wild boar were inoculated with the suspension of $M$. bovis BCG to a final concentration $10^{6} \mathrm{CFU} / \mathrm{g}$. The artificially contaminated samples were separated into two batches, the first left at room temperature for four hours and the second being frozen at $-20^{\circ} \mathrm{C}$ for one month in order to verify the viability of mycobacteria in frozen feces. The artificially infected samples were then decontaminated using two decontamination methods and cultured using two different culture media, in order to determine the more efficient culture method.

\section{Decontamination with NALC-NaOH and culture in Coletsos medium.}

Approximately $1 \mathrm{~g}$ of experimentally contaminated feces was placed in a $50-\mathrm{mL}$ Falcon tube complemented with PBS up to $5 \mathrm{~mL}$. An equal volume of $\mathrm{N}$-acetyl-Lcysteine-sodium hydroxide (NALC-NaOH) was added and the tube was vortexed and incubated for $15 \mathrm{~min}$ at room temperature with continuous agitation. The remaining 
volume was completed to $50 \mathrm{~mL}$ with a neutralization solution based on a phosphate buffer and centrifuged at 3,000 $\times \mathrm{g}$ for $20 \mathrm{~min}$. The supernatant was decanted, $1 \mathrm{~mL}$ of sterile PBS was added and $250 \mu \mathrm{L}$ of the pellet-PBS suspension was inoculated, in triplicate, in Coletsos medium (bioMérieux, Craponne, France). PBS and noninoculated feces were used in parallel as controls for culture to survey for crosscontamination and all tubes were incubated at $37^{\circ} \mathrm{C}$.

Decontamination with chlorhexidine $1 \%$ and culture in MOD9 medium. The MOD9 culture medium was prepared as previously described ${ }^{29,30}$. Briefly, MOD 9 culture medium incorporates for a total volume of $1.000 \mathrm{~mL}, 5 \mathrm{~g}$ of lecithin, $19 \mathrm{~g}$ of Middlebrook $7 \mathrm{H} 10$ powder, one gram of yeast extract, two grams of glucose, one gram of pancreatic digest of casein, $5 \mathrm{~mL}$ of glycerol and $2 \mathrm{~mL}$ of Tween 80. All compounds were dissolved in $669 \mathrm{~mL}$ of distilled water and the mixture was autoclaved at $121^{\circ} \mathrm{C}$ for $20 \mathrm{~min}$. Then $150 \mathrm{~mL}$ of lamb serum, $100 \mathrm{~mL}$ OADC (oleic acid albumin, dextrose, catalase), $20 \mathrm{~mL}$ of growth supplement (Becton Dickinson), $50 \mathrm{~mL}$ of antibiotic mixture solution (5 mg of vancomycin, 6,000 units of polymyxin B, $600 \mathrm{mg}$ of amphotericin B, $2,400 \mathrm{mg}$ of nalidixic acid, $600 \mathrm{mg}$ of trimethoprim and $600 \mathrm{mg}$ of azlocillin), $4 \mathrm{~mL}$ of red food colouring and $0.1 \mathrm{~g}$ of ascorbic acid were added after the medium was cooled to $55^{\circ} \mathrm{C}$. This culture medium has been specially designed for the culture-based recovery of mycobacteria including Mycobacterium tuberculosis complex. Decontamination with $1 \%$ chlorhexidine was performed according to ${ }^{46}$ modified by adding filtration as described below. About $1 \mathrm{~g}$ of experimentally contaminated feces was deposited into a $50 \mathrm{~mL}$-tube containing 3-mm sterile glass beads, and the volume was completed to $5 \mathrm{~mL}$ with sterile PBS. The tube was vortexed and the suspension was filtered with $40 \mu \mathrm{m}$ cell strainer to remove fibers and other large fecal particles. Two $\mathrm{mL}$ of this filtrate were mixed with a triple 
volume of $1 \%$ chlorhexidine, vortexed and incubated for $15 \mathrm{~min}$ at room temperature with continuous agitation. The remaining volume was completed to $50 \mathrm{~mL}$ with PBS and centrifuged at $3000 \mathrm{Xg}$ for $20 \mathrm{~min}$. The supernatant was then decanted, $1 \mathrm{~mL}$ of sterile PBS was added, and $100 \mu \mathrm{L}$ of the pellet-PBS suspension were inoculated, in duplicate, in MOD9 medium. PBS and non-inoculated feces were used in parallel as a negative control for culture to survey for cross contamination, and all plates were incubated at $37^{\circ} \mathrm{C}$. This method was further used on to decontaminate and inoculate 99 wild boar feces samples, including 79 samples collected at military camp A, and 20 samples collected at military camp B.

Artificially contaminated feces culture. The cultures were observed regularly and M. bovis strain BCG colonies were counted and identified with the matrix-assisted laser desorption/ionization time-of-flight mass spectrometry (MALDI-TOF-MS) on a Microflex spectrometer (Bruker Daltonics, Bremen, Germany) ${ }^{47,48}$. Briefly, one loopfull of colonies was removed from the culture and directly applied to the plate and mixed with matrix. For each colony, three different spots were deposited on the mass spectrometer plate and the manipulation was repeated after subculture. The protein profiles were visualized using FlexControl 3.3 software (Bruker Daltonik) and analyzed by the program FlexAnalysis 3.3 (Bruker Daltonik GmbH, Bremen, Germany).

Wild boar feces culture. After four months incubation at $37^{\circ} \mathrm{C}$, colonies observed were subcultured onto the same culture medium and incubated at the same temperature. An initial identification was carried out with MALDI-TOF-MS as previously described ${ }^{47,48}$. Colonies which remained not identified by MALDI-TOFMS, were stained by the Ziehl-Neelsen method and examined by using light microscopy. Ziehl-Neelsen-positive colonies were subsequently identified by 
275 sequencing PCR-amplified DNA. Genomic DNA was extracted using BIOROBOT

276 EZ1 and the Qiagen Genomic DNA Extraction kit (Qiagen, Courtaboeuf, France) as

277 previously described ${ }^{49}$. Sterile PBS was used as a negative control for extraction.

278 The 16S rRNA gene PCR amplification and sequencing were performed using the

$279 \mathrm{fD} 1 / \mathrm{rP} 2$ primer pair as previously described ${ }^{50}$. The partial rpoB gene PCR

280 amplification and sequencing were performed using the Myco F/Myco R primer pair

51. Sequencing was carried-out using the Big Dye Terminator version 3.1 kit (Perkin-

282 Elmer) and the resulting products were recorded with the Biosystems ABI Prism

3130xl as described by the manufacturer (Applied Biosystems, Massachusetts,

USA). The obtained nucleotide sequences were assembled using Chromas Pro

285 software, version 1.7 (Technelysium Pty Ltd., Tewantin, Australia) and compared to the GenBank database by similarity search using the BLASTN program 


\section{Declarations}

\section{Acknowledgements}

The authors thank the hunters of the military hunting companies of Canjuers and Carpiagne for their very efficient assistance in collecting the samples. This study was supported by IHU Méditerranée Infection, Marseille, France and by the French Government under the «Investissements d'avenir» (Investments for the Future) program managed by the Agence Nationale de la Recherche (ANR, fr: National Agency for Research), (reference: Méditerranée Infection 10-IAHU-03). This work was supported by Région Provence Alpes Côte d'Azur and European funding FEDER PRIMI. M. Fellag benefits a PhD grant from Fondation Méditerranée Infection, Marseille, France. The authors acknowledge Olga Cusack for her expert assistance in editing the manuscript.

\section{Competing interests}

The authors declare that they have no competing interests

\section{Ethics approval and consent to participate}

No Ethics or use committee approval was required since the samples of faeces were collected at a slaughterhouse, on wild boars already killed during hunting according to procedures officially approved by the French authorities (rifle shooting with large caliber bullets) and by authorized hunters. The hunt was not organized for the purpose of sample collection; no animal was killed in the perspective of the present study.

\section{Consent to publish}

Not applicable.

\section{Availability of data and materials}


bioRxiv preprint doi: https://doi.org/10.1101/670166; this version posted June 13,2019 . The copyright holder for this preprint (which was not certified by peer review) is the author/funder, who has granted bioRxiv a license to display the preprint in perpetuity. It is made available under aCC-BY-NC 4.0 International license.

314 All data generated or analysed during this study are included in this article. 


\section{References:}

1. Office International des Epizooties (OIE) bovine tuberculosis. In OIE Terrestrial Manual. Paris, France: OIE; 2009. Chapter 2.4.3.

2. Gortázar, C. et al. The status of tuberculosis in European wild mammals. Mammal Rev. 42, 193-206 (2012).

3. Palmer, M. V. Mycobacterium bovis: characteristics of wildlife reservoir hosts. Transbound. Emerg. Dis. 60 Suppl 1, 1-13 (2013).

4. Reveillaud, E. et al. Infection of wildlife by Mycobacterium bovis in France assessment through a national surveillance system, Sylvatub. Front. Vet. Sci. 5, (2018).

5. Delahay, R. J., Cheeseman, C. L. \& Clifton-Hadley, R. S. Wildlife disease reservoirs: the epidemiology of Mycobacterium bovis infection in the European badger (Meles meles) and other British mammals. Tuberculosis 81, 43-49 (2001).

6. Boniotti, M. B. et al. Detection and Molecular Characterization of Mycobacterium microti Isolates in Wild Boar from Northern Italy. J. Clin. Microbiol. 52, 2834-2843 (2014).

7. García-Jiménez, W. L. et al. Comparative pathology of the natural infections by Mycobacterium bovis and by Mycobacterium caprae in wild boar (Sus scrofa). Transbound. Emerg. Dis. 60, 102-109 (2013).

8. García-Jiménez, W. L. et al. Non-tuberculous mycobacteria in wild boar (Sus scrofa) from Southern Spain: epidemiological, clinical and diagnostic concerns. Transbound. Emerg. Dis. 62, 72-80 (2015). 
9. Machackova, M. et al. Wild boar (Sus scrofa) as a possible vector of mycobacterial infections: Review of literature and critical analysis of data from Central Europe between 1983 to 2001. ResearchGate 48, 51-65 (2003).

10. Trcka, I. et al. Mycobacterial infections in European wild boar (Sus scrofa) in the Czech Republic during the years 2002 to 2005. ResearchGate 51, (2006).

11. García-Agudo, L \& García-Martos, P. Clinical significance and antimicrobial susceptibility of rapidly growing mycobacteria. Sci. Microb. Pathog. Commun. Curr. Res. Technol. Adv. 77, 363-377 (2011).

12. Van der Werf, M. J. et al. Inventory study of non-tuberculous mycobacteria in the European Union. BMC Infect. Dis. 14, 62 (2014).

13. Santos, N. et al. Patterns of Mycobacterium tuberculosis-complex excretion and characterization of super-shedders in naturally-infected wild boar and red deer. Vet. Res. 46, 129 (2015).

14. Ghodbane, R. et al. M. Long-term survival of tuberculosis complex mycobacteria in soil. Microbiol. Read. Engl. 160, 496-501 (2014).

15. Drancourt, M. Looking in amoebae as a source of mycobacteria. Microb. Pathog. 77, 119-124 (2014).

16. Wilkins, M. J. et al. Human Mycobacterium bovis Infection and Bovine Tuberculosis Outbreak, Michigan, 1994-2007. Emerg. Infect. Dis. 14, 657-660 (2008).

17. Gw, De. L. et al. Tuberculosis in free-ranging wildlife: detection, diagnosis and management. Rev. Sci. Tech. Int. Off. Epizoot. 21, 317-334 (2002).

18. Pate, M. et al. Mycobacterium spp. in wild game in Slovenia. Vet. J. Lond. Engl. 1997 208, 93-95 (2016). 
19. Zanella, G. et al. Mycobacterium bovis in wildlife in france. J. Wildl. Dis. 44, 99108 (2008).

20. Oliveira, E. M. de D. et al. Comparison of methods for mycobacteria isolation from swine feces. Braz. J. Microbiol. 38, 687-692 (2007).

21. Whipple, D. L., Callihan, D. R. \& Jarnagin, J. L. Cultivation of Mycobacterium paratuberculosis from bovine fecal specimens and a suggested standardized procedure. J. Vet. Diagn. Investig. Off. Publ. Am. Assoc. Vet. Lab. Diagn. Inc 3, 368-373 (1991).

22. Michelet, L. et al. Mycobacterium bovis Infection of Red Fox, France. Emerg. Infect. Dis. 24, 1150-1153 (2018).

23. Asmar, S. \& Drancourt, M. Rapid culture-based diagnosis of pulmonary tuberculosis in developed and developing countries. Front. Microbiol. 6, (2015).

24. Hars, J., Richomme, C., Rivière, J., Faure, E. \& Boschiroli, M. L. Dix années de surveillance de la tuberculose bovine dans la faune sauvage française et perspectives. Bull. Epid. Santé anim. Alim, 52, 2-6. (2012).

25. Chevalier, F. et al. Découverte d'un sanglier infecté par M. bovis en Sologne. Bull Epid Santé anim Alim 72:12-16. (2015).

26. Richomme, C. et al. Exposure of Wild Boar to Mycobacterium tuberculosis Complex in France since 2000 Is Consistent with the Distribution of Bovine Tuberculosis Outbreaks in Cattle. PLOS ONE 8, e77842 (2013).

27. Fellag, M. et al. Draft Genome Sequence of "Nocardia suismassiliense" Strain S137 (CSUR P4007). Genome Announc. 6, e00212-18 (2018).

28. Office International des Epizooties (OIE) Paratuberculosis (Johne's disease). In OIE Terrestrial Manual. Paris, France: OIE; 2014. Chapter 2.1.15. 
29. Ghodbane, R., Raoult, D. \& Drancourt, M. Dramatic reduction of culture time of Mycobacterium tuberculosis. Sci. Rep. 4, 4236 (2014).

30. Asmar, S. et al. A Novel Solid Medium for Culturing Mycobacterium tuberculosis Isolates from Clinical Specimens. J. Clin. Microbiol. 53, 2566-2569 (2015).

31. Tessema, B., Beer, J., Emmrich, F., Sack, U. \& Rodloff, A. C. Rate of recovery of Mycobacterium tuberculosis from frozen acid-fast-bacillus smear-positive sputum samples subjected to long-term storage in Northwest Ethiopia. J. Clin. Microbiol. 49, 2557-2561 (2011).

32. Witkowski, L. et al. Evidence of low prevalence of mycobacterial lymphadenitis in wild boars (Sus scrofa) in Poland. Acta Vet. Scand. 59, 9 (2017).

33. Hachem, R. et al. Cutaneous and pulmonary infections caused by Mycobacterium vaccae. Clin. Infect. Dis. Off. Publ. Infect. Dis. Soc. Am. 23, 173-175 (1996).

34. Lowry, C. A. et al. Identification of an immune-responsive mesolimbocortical serotonergic system: Potential role in regulation of emotional behavior. Neuroscience 146, 756-772 (2007).

35. Weng, H., Huang, J.-Y., Meng, X.-Y., Li, S. \& Zhang, G.-Q. Adjunctive therapy of Mycobacterium vaccae vaccine in the treatment of multidrug-resistant tuberculosis: A systematic review and meta-analysis. Biomed. Rep. 4, 595-600 (2016).

36. Kazumi, Y. \& Mitarai, S. The evaluation of an identification algorithm for Mycobacterium species using the 16S rRNA coding gene and rpoB. Int. J. Mycobacteriology 1, 21-28 (2012).

37. Aranaz, A. et al. Mycobacterium peregrinum infection in farmed European tench (Tinca tinca L.). Vet. Microbiol. 131, 393-399 (2008). 
38. Nagao, M. et al. Surgical site infection due to Mycobacterium peregrinum: a case report and literature review. Int. J. Infect. Dis. IJID Off. Publ. Int. Soc. Infect. Dis. 13, 209-211 (2009).

39. Lamy, B., Marchandin, H., Hamitouche, K. \& Laurent, F. Mycobacterium setense sp. nov., a Mycobacterium fortuitum-group organism isolated from a patient with soft tissue infection and osteitis. Int. J. Syst. Evol. Microbiol. 58, 486-490 (2008).

40. Toro, A., Adekambi, T., Cheynet, F., Fournier, P.-E. \& Drancourt, M. Mycobacterium setense infection in humans. Emerg. Infect. Dis. 14, 1330-1332 (2008).

41. Shojaei, H. et al. First Report on Isolation and Molecular Characterization of Clinical Mycobacterium setense Isolates in Asia. Jpn. J. Infect. Dis. 64, 234-236. (2011).

42. Rech, G. et al. Draft Genome Sequences of Mycobacterium setense Type Strain DSM-45070 and the Nonpathogenic Strain Manresensis, Isolated from the Bank of the Cardener River in Manresa, Catalonia, Spain. Genome Announc. 3, e01485-14 (2015).

43. Everest, G. J., Cook, A. E., le Roes-Hill, M. \& Meyers, P. R. Nocardia rhamnosiphila sp. nov., isolated from soil. Syst. Appl. Microbiol. 34, 508-512 (2011).

44. Kaba, M., Davoust, B., Marié, J.-L. \& Colson, P. Detection of hepatitis E virus in wild boar (Sus scrofa) livers. Vet. J. 186, 259-261 (2010).

45. Colson, P. et al. Pig Liver Sausage as a Source of Hepatitis E Virus Transmission to Humans. J. Infect. Dis. 202, 825-834 (2010).

46. Asmar, S. \& Drancourt, M. Chlorhexidine decontamination of sputum for culturing Mycobacterium tuberculosis. BMC Microbiol. 15, 155 (2015). 
47. Seng, P. et al. Ongoing Revolution in Bacteriology: Routine Identification of Bacteria by Matrix-Assisted Laser Desorption Ionization Time-of-Flight Mass Spectrometry. Clin. Infect. Dis. 49, 543-551 (2009).

48. Zingue, D., Flaudrops, C. \& Drancourt, M. Direct matrix-assisted laser desorption ionisation time-of-flight mass spectrometry identification of mycobacteria from colonies. Eur. J. Clin. Microbiol. Infect. Dis. Off. Publ. Eur. Soc. Clin. Microbiol. 35, 1983-1987 (2016).

49. Djouadi, L. N. et al. Mycobacterium icosiumassiliensis sp. nov., a New Member in the Mycobacterium terrae Complex Isolated from Surface Water in Algeria. Curr. Microbiol. 73, 255-264 (2016).

50. Drancourt, M. et al. 16S Ribosomal DNA Sequence Analysis of a Large Collection of Environmental and Clinical Unidentifiable Bacterial Isolates. J. Clin. Microbiol. 38, 3623-3630 (2000).

51. Adékambi, T., Colson, P. \& Drancourt, M. rpoB-based identification of nonpigmented and late-pigmenting rapidly growing mycobacteria. J. Clin. Microbiol. 41, 5699-5708 (2003). 
Table 1. Non-tuberculous mycobacteria isolated form wild-boar feces and identified by rpoB sequencing; along with Nocardia species identified by $16 \mathrm{~S}$ rRNA sequencing.

\begin{tabular}{|c|c|c|c|c|}
\hline $\begin{array}{l}\text { Bacterial } \\
\text { species }\end{array}$ & $\begin{array}{l}\text { Number } \\
\text { positive }\end{array}$ & $\begin{array}{l}\text { Match of } \\
\text { GenBank (\%) }\end{array}$ & $\begin{array}{l}\text { GenBank } \\
\text { accession }\end{array}$ & MALDI-TOF score \\
\hline $\begin{array}{l}\text { Mycobacterium } \\
\text { peregrinum }\end{array}$ & 3 & 99 & AY147166.1 & $\begin{array}{l}\text { Identified with a } \\
\text { high score (2.033) }\end{array}$ \\
\hline $\begin{array}{l}\text { Mycobacterium } \\
\text { setense }\end{array}$ & 1 & 100 & HM807426.1 & $\begin{array}{l}\text { Identified with a } \\
\text { middle score (1.88) }\end{array}$ \\
\hline $\begin{array}{l}\text { Mycobacterium } \\
\text { vaccae }\end{array}$ & 1 & 100 & CP011491.1 & $\begin{array}{l}\text { Identified with a } \\
\text { high score (1.915) }\end{array}$ \\
\hline $\begin{array}{l}\text { Nocardia } \\
\text { rhamnosiphila }\end{array}$ & 1 & 100 & KC417288.1 & Not identified \\
\hline $\begin{array}{l}\text { Nocardia } \\
\text { Strain S-137 }\end{array}$ & 1 & 98 & CP022088.2 & Not identified \\
\hline
\end{tabular}

